\title{
Erratum to: Numerical investigation of two-sided reinforced laminated glass beams in statically indeterminate systems
}

\author{
Kenny Martens · Robby Caspeele · Jan Belis
}

Published online: 30 June 2016

(C) Springer International Publishing Switzerland 2016

Erratum to: Glass Struct. Eng. DOI 10.1007/s40940-016-0005-6

Both co-authors, Robby Caspeele and Jan Belis, were omitted from this article. Please see below for their full affiliations.

The online version of the original article can be found under doi:10.1007/s40940-016-0005-6.

K. Martens $(\varangle) \cdot$ R. Caspeele $\cdot$ J. Belis

Ghent University, Technologiepark Zwijnaarde 904,

9052 Zwijnaarde, Belgium

e-mail: kenny.martens@ugent.be

R. Caspeele

e-mail: robby.caspeele@ugent.be

J. Belis

e-mail: jan.belis@ugent.be 